DOE/MC/29103-95/C0459

Lightguide-Coupled Sensor for In-Situ Radiation Monitoring

Authors:

S. E. Reed

J. W. Berthold

ConF-9506183-1

\title{
Contractor:
}

Babcock \& Wilcox

1562 Beeson Street

Alliance, Ohio 44601-9110

Contract Number:

DE-AC21-92MC29103

Conference Title:

Optics for Environmental and Public Safety

Conference Location:

Munich Bavaria, FRG

\section{Conference Dates:}

June $19-23,1995$

\section{Conference Sponsor:}

The International Society for Optical Engineering 


\section{DISCLAIMER}

This report was prepared as an account of work sponsored by an agency of the United States Government. Neither the United States Government nor any agency thereof, nor any of their employees, makes any warranty, express or implied, or assumes any legal liability or responsibility for the accuracy, completeness, or usefulness of any information, apparatus, product, or process disclosed, or represents that its use would not infringe privately owned rights. Reference herein to any specific commercial product, process, or service by trade name, trademark, manufacturer, or otherwise does not necessarily constitute or imply its endorsement, recommendation, or favoring by the United States Government or any agency thereof. The views and opinions of authors expressed herein do not necessarily state or reflect those of the United States Government or any agency thereof.

This report has been reproduced directly from the best available copy.

Available to DOE and DOE contractors from the Office of Scientific and Technical Information, 175 Oak Ridge Turnpike, Oak Ridge, TN 37831; prices available at (615) 576-8401.

Available to the public from the National Technical Information Service, U.S. Department of Commerce, 5285 Port Royal Road, Springfield, VA 22161; phone orders accepted at (703) 487-4650. 


\section{DISCLAIMER}

Portions of this document may be illegible in electronic image products. Images are produced from the best available original document. 


\title{
LIGHTGUIDE-COUPLED SENSOR FOR IN SITU RADIATION MONITORING
}

\author{
S. E. Reed and J. W. Berthold \\ Babcock \& Wilcox — Research and Development Division \\ 1562 Beeson Street, Alliance, OH 44601
}

\begin{abstract}
Under contract to the Morgantown (WV) Energy Technology Center for the Office of Technology Development (EM-50), U.S. Department of Energy, we are developing a multi-point radiation monitoring system for long-term, continuous monitoring of radiation levels in the vadose zone of radioactive waste sites. The system is based on gamma detection with a lightguide-coupled scintillator built into a probe buried in the ground. The lightguide transmits the visible light pulses produced by the scintillator to the surface where detection and signal multiplexing take place. The system is to be capable of monitoring large numbers of such passive probes which are to be permanently installed throughout the waste site. We have recently completed tests of a prototype single-probe system. In this paper, we report on the development and testing of the single-probe system.
\end{abstract}

\section{INTRODUCTION}

The long-term monitoring of the vadose zone of a hazardous waste site for migration of radionuclides requires installation of radiation sensors at a large number of subsurface locations. Sensors which can be lowered into boreholes to make radiation measurements are available, but are relatively complex and expensive, and generally are not suitable for long-term installation underground. The existing devices normally incorporate a transduction element (e.g., a scintillator or solid-state device) to convert ionizing radiation to an electrical or an electromagnetic (light) emission. In the case of electromagnetic transducers, the emitted light or photons are further converted to an electrical quantity via an attached photoelectronic device (e.g., an electrically powered photomultiplier tube attached directly to a scintillation crystal). Each subsoil point to be monitored must then be equipped with a separate sensor containing the transducer and electronic component. The cost of installing a large number of these radiation sensors in the vadose zone of a waste site can be cost-prohibitive and the need for high reliability of the electronic devices underground over a 30 -year period would require a significant maintenance budget and costly periodic sensor replacement.

The problem of measuring low-level radiation at a large number of locations over a long period of time is amenable to a solution based on fiber optic technology. The concept developed employs an in-ground measurement probe which contains a scintillator coupled to a lightguide such as an optical fiber. Because of the limited range of alpha $(<0.015 \mathrm{~cm})$ and beta $(<1.5$ $\mathrm{cm}$ ) radiation in soil, the scintillator is designed to respond primarily to gamma radiation, with a range on the order of a meter. In one concept, shown in Figure 1, optical fibers from a large number of in-ground probes could be routed to a small building on the surface containing multiplexing equipment, a single opto-electronic detector, and a personal computer. In an alternate approach, the scintillators could be coupled to large diameter lightguides and the detectors located at the end of each probe on the surface. No subsurface electrical power is required nor is any generated. Gamma radiation absorbed by the in-ground scintillator generates optical photons which are coupled into the lightguide, conveyed to the surface, and detected as an indication of the instantaneous subsurface radiation level. The system is to be capable of continuous, unattended monitoring of an array of in-ground detectors to provide an indication of radionuclide migration. The computer is intended to continuously map the distribution of radionuclides and to be capable of being interrogated from a remote location.

The in-ground probes are to be configured to geometrically resemble cone penetrometers (CPT) or penetrometer-based sampling tools and thus can be installed into the ground to depths of up to 50 meters using conventional CPT trucks and methods. Alternatively, the probe assembly could be installed in existing sampling wells, if available. Figure 2 is a conceptual drawing of the system installed at a waste site for long-term monitoring. The use of the CPT geometry provides a proven method for relatively rapid inexpensive installation of multiple probes compared to the cost of drilling and casing a comparable number of wells. The use of the penetrometer eliminates the cuttings normally associated with drilled wells, and thus minimizes worker exposure and eliminates the cost of cutting disposal. The penetrometer approach also has the advantage of 
producing minimal cross-contamination; the "smearing" which can result from conventional well drilling can spread contamination from one stratum to another and invalidate measurements. A large number of in-ground passive probes can be multiplexed to a single, above-ground opto-electronics unit, which would provide for detection and readout of any long-term changes in the distribution of the radionuclides in the vadose zone. Because the in-ground components (the scintillator and fiber optic cable) are passive and require no electrical power, the impediments to long life and maintenance-free operation are removed.

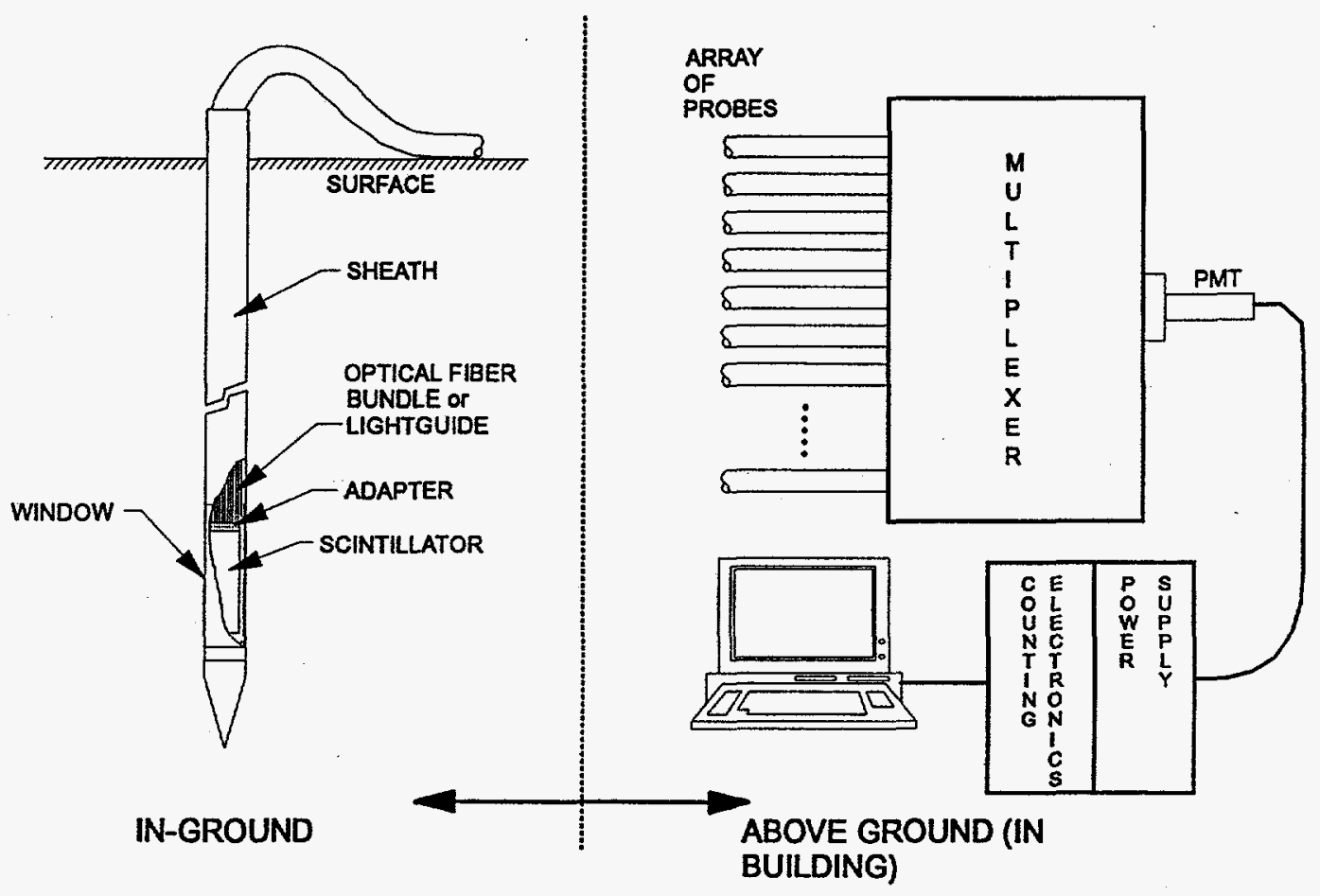

Fig. 1. System concept

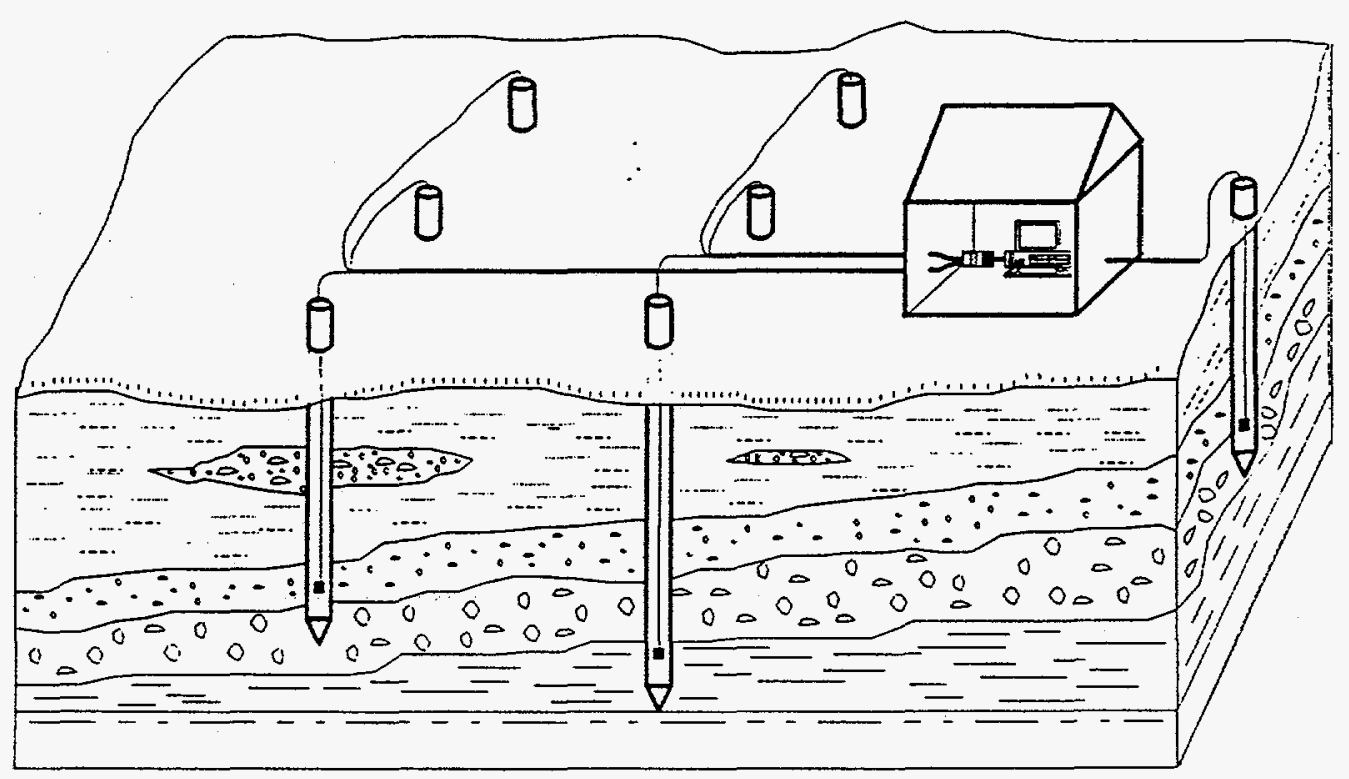

Fig. 2. Conceptual drawing of an installed system 


\section{BACKGROUND AND PROBLEM DEFINITION}

Our objectives have been to configure a probe with the highest sensitivity to gamma radiation and the lowest optical losses, within the available CPT envelope, using commercially available components, at the lowest possible cost. In addition, it was judged highly desirable that the resultant design maintain the potential for energy discrimination which might be needed for nuclide identification or background compensation. The approach was to identify the nuclides of concern at DOE sites (to determine the required detection range of gamma energies and to permit quantitative estimates of performance), to optimize each component of the probe in signal path sequence between the soil and the opto-electronics, and to assess their individual loss characteristics and their combined behavior in a probe. The overall performance of the probe could then be estimated to determine the volume of soil which could be monitored by the probe and the expected output optical pulse heights and rates for each of the nuclides of concern.

A total of 23 radionuclides were identified as occurring with significant frequency and activity on DOE lands, including natural decay products and fission fragments. Decay chain relationships were examined for each of these. For 20 of these nuclides, viable gamma monitoring approaches are available based on direct monitoring, short-lived daughters or known isotopic ratios.

The radionuclides occurring most frequently and exhibiting the highest activities are isotopes of uranium (U-234, U-235, and U-238), radium ( $\mathrm{Ra}-224, \mathrm{Ra}-226$, and $\mathrm{Ra}-228$ ), and thorium (Th-228, Th-230, and Th-232). These are all natural decay products from the Thorium ( $4 n)$, Uranium $(4 n+2)$ and Actinium $(4 n+3)$ series. For the decay of some of the radionuclides, e.g., U-234 to Th-230 to Ra-226, there are no short-lived daughters and these nuclides will not be at equilibrium in the monitoring period; the activity of these nuclides will depend primarily on their initial concentration rather than on decay of a parent. The nuclides with long-lived daughters must thus be monitored by direct detection, or must be inferred from known isotopic ratios.

Other radionuclides identified to occur less frequently include both natural decay products and fission fragments. The natural decay products include nuclides from the decay chains such as Ac-227 $(4 n+3)$ and $\mathrm{Pb}-210(4 n+2)$ plus their daughters. The fission fragments identified in the compilation include Cobalt-60, Strontium-90, and Cesium-137; Co-60 and Cs-137 have readily detectable high-yield energetic gamma lines (1173/1333 KeV and $662 \mathrm{KeV}$, respectively). Sr-90 and its short-lived daughter Y-90 decay by beta with no gamma emission and are undetectable with this system. Other radionuclides which are likely to occur at one or more sites are H-3 (Tritium), Tc-99, Ru-105, and Ce-144. Of these, tritium and Tc-99 will be undetectable with this system because of the low energy of its beta decay (18.6 KeV maximum).

A soil model was developed and used to examine the effects of soil density and degree of saturation on the gamma flux through the scintillator volume. While variations in soil density can produce changes in gamma flux of $30 \%$ or more, changes in soil density large enough to cause concern are not judged likely barring major disturbances in the subsurface soils or major changes in surface conditions. Periodic or seasonal variations in the degree of saturation of the soil within the monitored volume are more likely. A variation of $50 \%$ in the soil water content (likely in some soils) will produce a $10 \%$ variation in the gamma flux and thus a 10\% change in the output of the monitor. The effective radius over which soil radionuclides contribute to the probe output was also calculated, and found to vary over a factor of three with energy within a given soil type, and also to have significant variations with soil density and degree of saturation. This may influence the system design but should not be an operational concern.

\section{DESIGN APPROACH}

The probe design included evaluation of scintillators, alternative methods of optical coupling, and evaluation of candidate lightguides.

\subsection{Scintillator}

Four classes of commercially available scintillators were evaluated for overall efficiency based on density, mass absorption characteristics, scintillation efficiency, and emission wavelength. Three alternative shape geometries were evaluated for collection efficiency and uniformity. Based on these evaluations, the preferred shape of the scintillator for the long-term postclosure radiation monitor is a large aspect ratio cylindrical scintillator of thallium-doped cesium iodide, CsI(TI). Thallium- 
doped sodium iodide, $\mathrm{NaI}(\mathrm{Tl})$, would be a potential alternative, although its shorter emission wavelength would result in increased spectral losses in the transmission fiber and its hygroscopic nature would require greater attention in the probe design and fabrication. Both conic and compound parabolic collector (CPC) geometries for the scintillator have greater collection efficiencies than the cylinder, but are highly non-uniform in response, precluding energy discrimination. Figure 3 shows that on an optical photon basis, CsI(Tl) has a higher overall efficiency than NaI(Tl), especially at energies higher than $300 \mathrm{KeV}$. This is due in large part to the fact that the emission wavelength of CsI(Tl) is longer than that of $\mathrm{NaI}(\mathrm{Tl}), 540 \mathrm{~nm}$ compared to $415 \mathrm{~nm}$. The average optical photon energy for CsI(Tl) is about $2.3 \mathrm{eV}$ while for $\mathrm{NaI}(\mathrm{Tl})$ it is about $3 \mathrm{eV}$, thus on an energy basis, the two are just about equal. The longer emission wavelength of CsI(Tl) provides a better match for the fiber, reducing spectral losses in transmission. The index of refraction of CsI(TI) is 1.80 . CsI(TI) is more rugged and less hygroscopic material than $\mathrm{NaI}(\mathrm{Tl})$, and thus more suitable for long-term in-ground application.

A parametric study using a soil model showed that for scintillators which absorb a significant fraction of incident gamma flux, increasing the length of the scintillator was more effective in increasing optical output than increasing its radius. This is so because increasing the length increases the total volume of soil which contributes to the incident gamma flux as well as the fraction absorbed, while increasing the radius only increases the fraction absorbed. Thus, the most obvious choice for the scintillator shape geometry is a cylinder with a relatively large aspect ratio (length/diameter). This geometry has the advantage of being relatively simple to manufacture and also is highly compatible with the geometry of the CPT. In addition to the cylinder, two other geometries - a conic taper and a CPC - were also evaluated to determine whether they could provide significant gains in collection efficiency compared to the cylindrical geometry. Both of these geometries have been used as non-imaging concentrators for solar energy applications and for coupling scintillation light into PMTs. In both these applications, the energy source has comparatively small divergence angles with large area and is coupled into a device with a large acceptance angle but small area. Our application is essentially the opposite: the scintillation events are nearly point source spherical emitters (small area, large angles) within the collector volume, to be coupled into a lightguide with a limited acceptance angle. The collector orientation is thus reversed from that normally encountered: the normal "input" end (the larger diameter) is connected to the lightguide, while the smaller "output" end is mirrored to feed light back towards the lightguide.

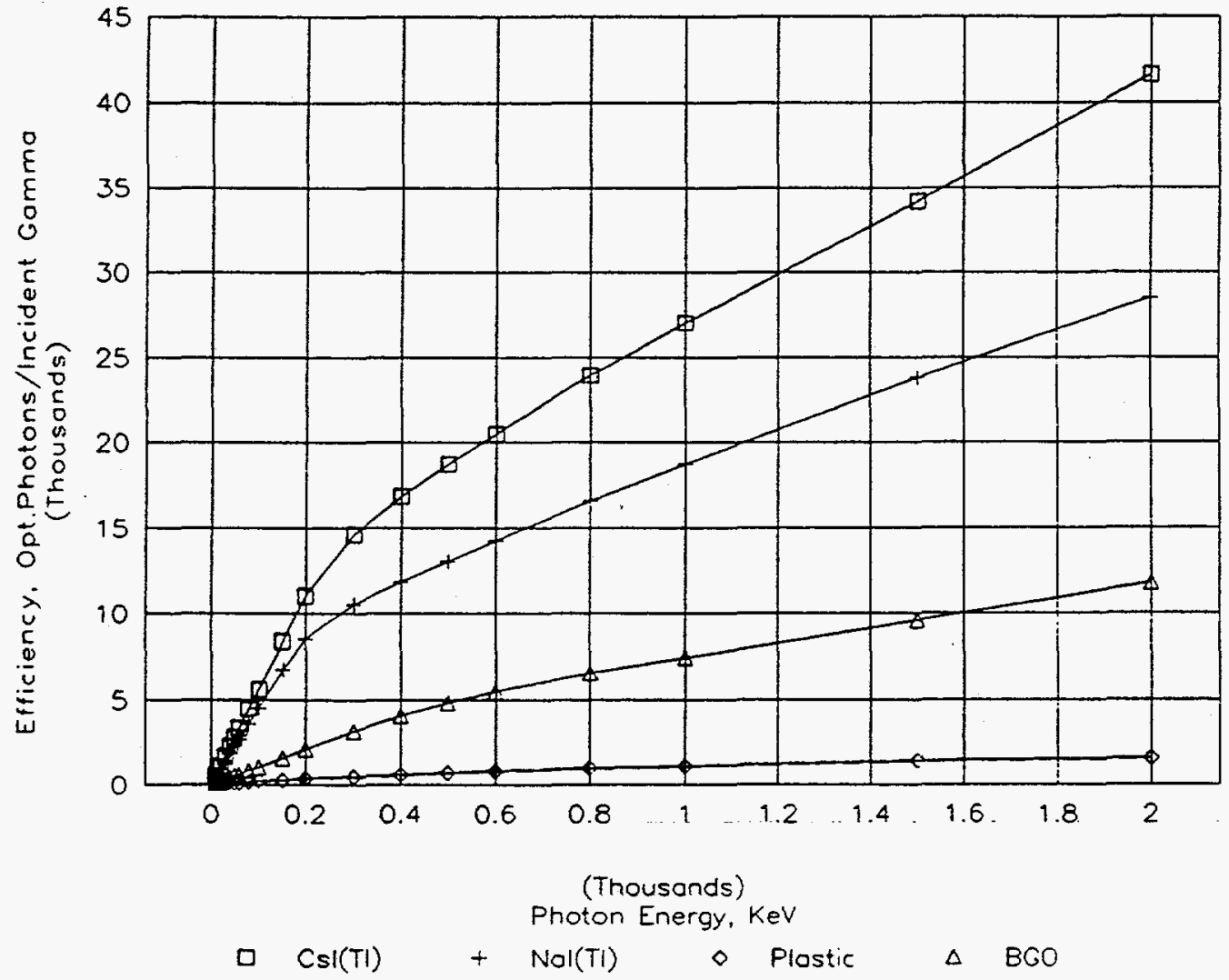

Fig. 3. Overall conversion efficiency versus energy $(0-2 \mathrm{MeV})$ (Each scintillator is $2.5 \mathrm{~cm}$ thick) 
To evaluate and compare the three scintillator shape geometries, ray tracing was used. The results from the ray-tracing analyses for the axial centerline locations are summarized in Figure 4 . These data show that the cylindrical scintillator is very uniform in its response, showing a maximum variation in energy output of only about $8 \%$ regardless of the axial position of the source, while the number of rays stays constant. The average number of reflections in the scintillator for rays which reach the image plane is only about 1.3 ; thus increasing the surface reflectivity of the scintillator from 0.87 to 0.93 would reduce the $8 \%$ variation with axial position, but would increase the energy output less than $10 \%$. The total energy collected in the scintillator is relatively low: about $0.5 \%$ of the emitted energy at $0.22 \mathrm{NA}$ and about $1.7 \%$ at $0.36 \mathrm{NA}$. With a cylindrical scintillator, the efficiency of the energy collection is primarily affected by the numerical aperture (NA) of the lightguide, and is proportional to the square of the NA. The uniformity of the response is not degraded by increases in NA.

The responses of both the conic and CPC scintillator shapes are peaked toward the small end (away from the lightguide). For the CPC, the energy collected from a scintillation event near the small end is more than 20 times that of an equal energy event near the large end. The response falls to a level at or below that of the cylindrical scintillator over nearly $2 / 3$ of the length. The collection efficiency of the CPC (into $0.36 \mathrm{NA}$ ) ranges from almost $30 \%$ at the small end to about $1.5 \%$ at the large end, with an average of $4.2 \%$. The average number of reflections within the scintillator is between 2 and 2.5 ; thus, increasing the reflectivity would increase the energy collected by about $18 \%$. The cone is less sharply peaked than the CPC, and the peak falls off less rapidly; less than $1 / 3$ of the length has a response at or below that of the cylinder. The collection efficiency of the cone (into $0.36 \mathrm{NA}$ ) ranges from $23 \%$ at the small end to $1.1 \%$ at the large end, with an average of $5.5 \%$. The rays are reflected 4 to 8 times within the cone, thus this geometry would benefit the most from an increase in surface reflectivity; such an increase would also increase the non-uniformity. An increase to 0.93 would increase the energy captured by nearly $50 \%$ at the end near the fiber and more than $75 \%$ at the far end.

After consideration of the results of the ray-trace analyses, we decided to use a cylindrically shaped scintillator because the small gains that might be achieved from other shapes did not outweigh the geometrical simplicity of the cylinder and the ease of coupling it to the lightguide. The major benefit of the cylindrical geometry is the uniformity of response, which permits spectroscopy and energy discrimination to be performed.

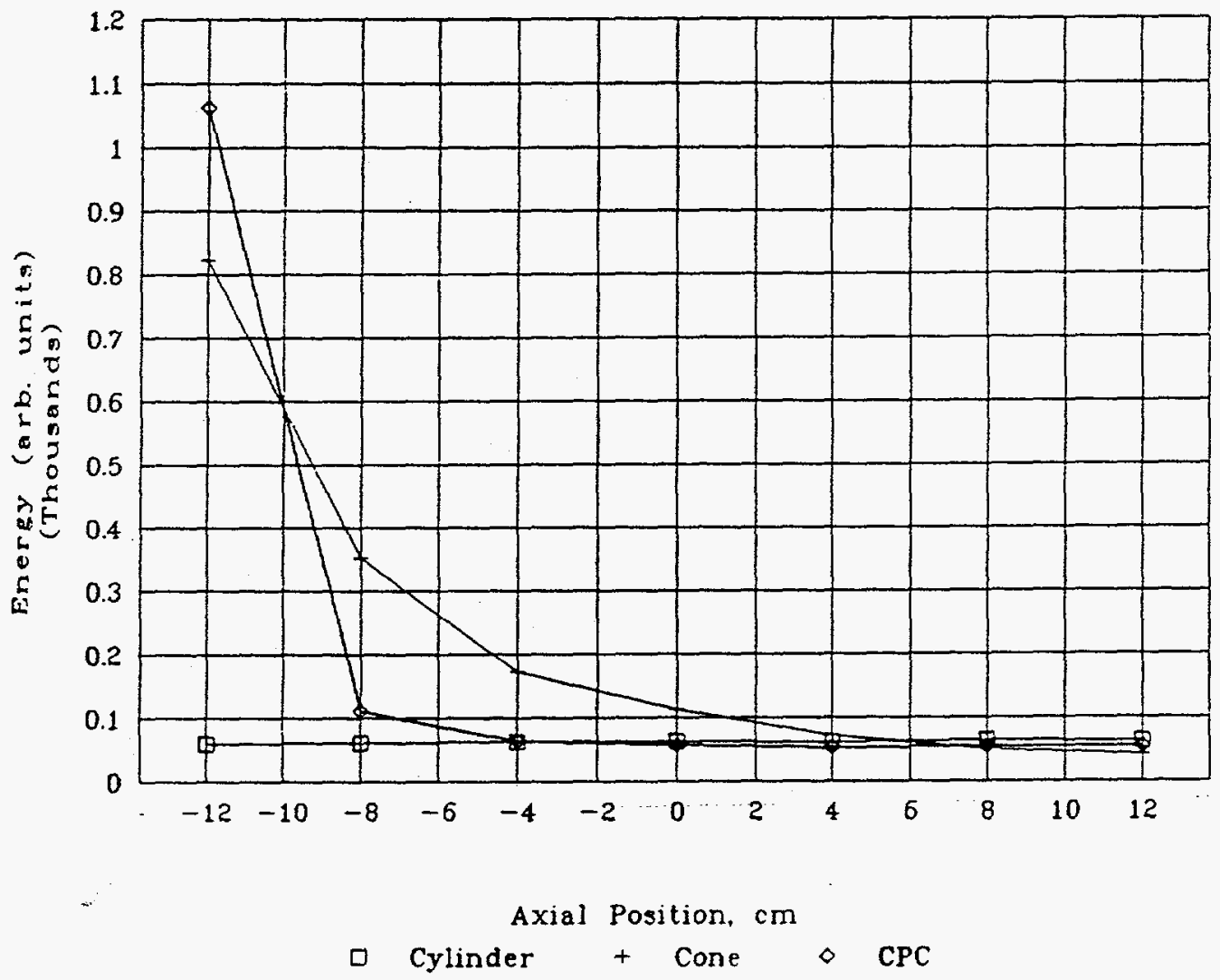

Fig. 4. Energy collection of alternative scintillator geometries 


\subsection{Coupling and Fiber Selection}

Conventional optical elements and several alternative methods of coupling a scintillator to a lightguide were evaluated to determine if it was possible to couple more light into the lightguide than would be coupled in by directly butt-coupling the lightguide to the scintillator. It was found that none of the means evaluated could improve on the butt-coupled geometry. Because of its simplicity and efficiency, the butt-coupled geometry is the preferred approach for the long-term post-closure radiation monitor.

Six commercially available candidate lightguides were evaluated for suitability as the means to couple the scintillator and detector. Based on all of the parameters investigated (cost, NA, spectral loss characteristics, and stem effect), the preferred lightguide for the long-term post-closure radiation monitor is made from a poly-methyl methacrylate (PMMA)/fluorinated polymer. Lightguides made from this material are readily available in relatively large diameters. To reduce the cost of the required large area, we used as an alternative, PMMA rods which could be clad with Teflon AF or air clad.

At large NAs and including the contribution from reflection, the total fraction of the emitted light which is captured is less than 10\%; for lightguides with NA in the range of 0.5 to 0.6 , the fraction is about $5 \%$ or less. The effective NA decreases with length as a result of the losses of higher order modes. The effective NA was measured for a number of available lightguide types and at several different lengths. The effective NA was determined by measuring the optical transmission of the lightguide using a collimated beam of light input to the test fibers over a range of angles. The results of these measurements are shown in Table 1. The data in Table 1 show that the plastic lightguides, while showing more NA dependence on length than the silica lightguides, have a substantial advantage in NA.

\subsection{Probe Design}

Based on the radionuclides identified and the analyses and tests described above, a prototype probe was designed for testing. The purpose of the testing of this probe is to validate the calculations and methods used to design and predict its performance, and to provide a demonstration of a single channel system in a short-term field test at a DOE site in actual contaminated soil.

The mechanical design of the probe is based on the dimensional envelope of a $10 \mathrm{~cm}^{2}$ cone penetrometer with a $3.65 \mathrm{~cm}$ outside diameter and a conventional 60-degree cone tip angle. As shown in Figure 5, the probe consists of a scintillation head housing the scintillator, a detection head housing the PMT and detection electronics, and several threaded extension sections for the push rods and lightguide. The scintillation head incorporates a $2.5 \mathrm{~cm}$ diameter by $25 \mathrm{~cm}$ long CsI(TI) scintillator inside a steel window section which extends slightly past the scintillator on both ends. In this design, the steel window material can easily carry a push force of 20 tons applied to the CPT tool, but because of the relatively short length of the

Table 1

\section{EFFECTIVE NA MEASUREMENTS}

$\begin{array}{lccc}\text { Lightguide Type } & \text { Length } & \text { Effective NA } & \text { Nominal NA } \\ \text { SiO/SiO } & & & \\ \text { SiO/SiO }_{2} & 51^{\prime \prime} & 0.21 & 0.22 \\ & 561^{\prime \prime} & 0.19 & 0.22 \\ \text { Plastic Clad SiO } & 120^{\prime \prime} & 0.37 & 0.36 \\ \text { Plastic Clad SiO } & 396^{\prime \prime} & 0.37 & 0.36 \\ & & & \\ \text { PMMA/Fluor. } & 117^{\prime \prime} & 0.45 & 0.50 \\ \text { PMMA/Fluor. } & 397^{\prime \prime} & 0.42 & 0.50 \\ & & & \\ \text { Styrene/PMMA } & 8^{\prime \prime} & 0.55 & 0.58 \\ \text { Styrene/PMMA } & 40^{\prime \prime} & 0.51 & 0.58 \\ \text { Styrene/PMMA } & 80^{\prime \prime} & 0.38 & 0.58\end{array}$


probe (about 2 meters), it will be fully assembled above ground prior to installation and testing. This will permit the probe to be moved between test locations, much like a survey tool. Probes intended for full-depth installations using a CPT truck will be assembled 1 meter at a time during the push, similar to CPT strings.

The optical photons from the scintillator are transmitted by a $2.5 \mathrm{~cm}$ diameter single air-clad PMMA lightguide directly butt-coupled to the scintillator. At the surface end of the probe, the extension sections are coupled to a detection head containing a $28 \mathrm{~mm}$ head-on PMT, a voltage divider base, a pre-amp, and pulse shaping electronics. The lightguide is directly butt-coupled to the PMT face.

The PMT is operated in the pulse mode with a cathode ground (positive high voltage). This mode of operation is consistent with either photon counting or spectroscopic analysis techniques. The PMT is magnetically shielded, and is shielded from background radiation (terrestrial and cosmogenic) using low radioactivity materials. Energy discrimination by pulse height analysis permits nuclide identification and background subtraction.

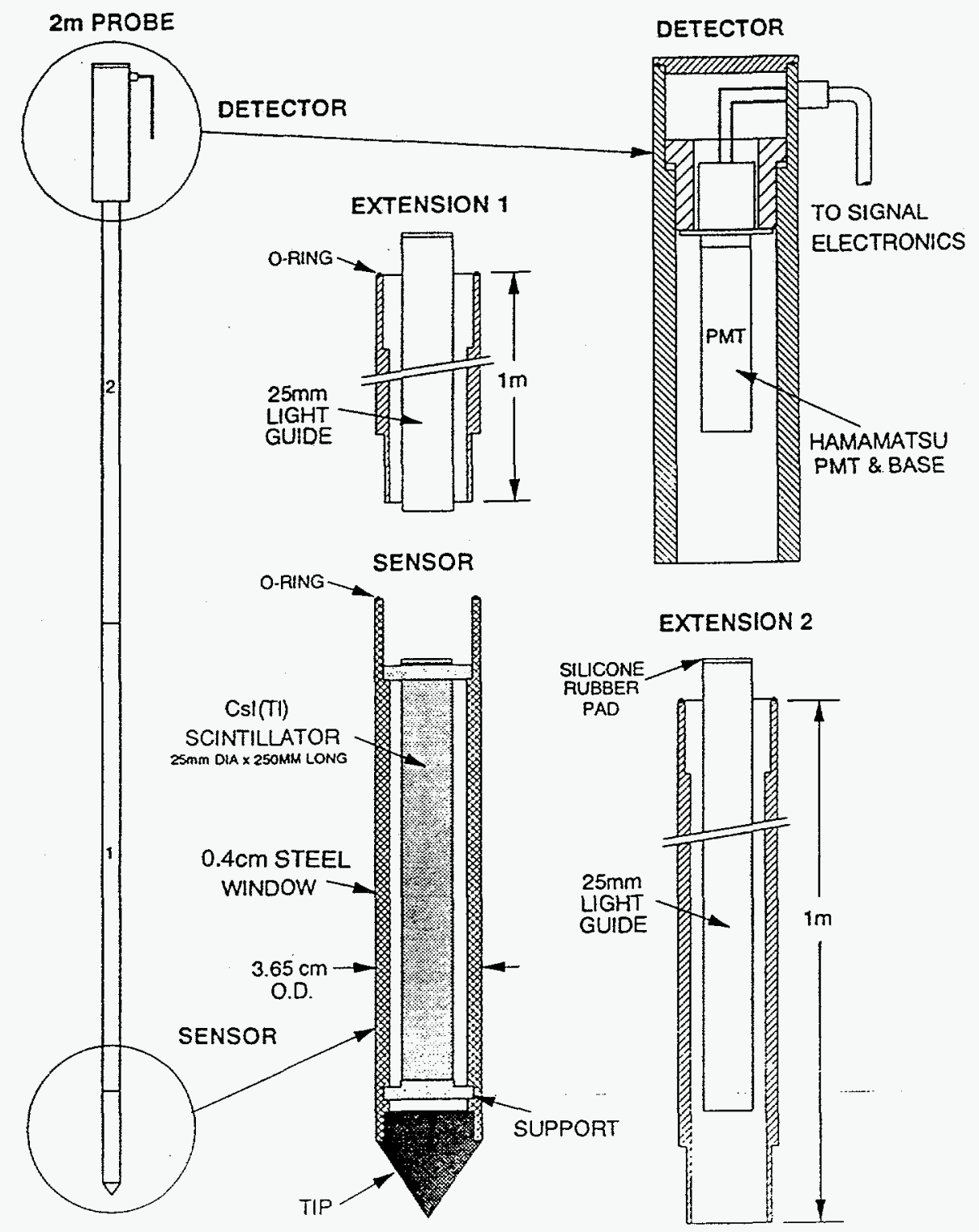

Fig. 5. Prototype probe design 


\section{LAB TESTS AND RESULTS}

The performance of the prototype design was estimated using a soil model for each of the radionuclides identified at an activity level of $5 \mathrm{pCi} / \mathrm{g}$, except the pure beta emitters $\mathrm{H}-3$ and Sr-90/Y-90. Direct gamma contributions and contributions from daughters in secular equilibrium were included (maximum of seven gamma lines). The analyses for all of the radionuclides were performed assuming a soil density of $1.2 \mathrm{~g} / \mathrm{cc}$, dry conditions. The analyses were then repeated assuming saturation conditions for four species (U-234, Pa-233, Cs-137, and Co-60) with a wide range of gamma energies (53 KeV to 1.333 $\mathrm{MeV}$ ) to ascertain the effect of water on the count rates. The analyses estimated both the number of optical photons per count and the number of counts per minute at the PMT.

A prototype probe of similar design to the one shown in Figure 5 was built and tested at Fernald, OH. A second probe (No. 2) containing a sodium iodide scintillator and PMT, but without a lightguide was also built and tested along with Probe No. 1 containing the lightguide. All of the data were analyzed using gamma spectroscopy. Comparisons can be made in the data from both probes to determine the effects of the lightguide on the probe performance. Figures 6 and 7 are data plots from each probe after insertion into the same radioactive soil sample. Note that both probes identify the presence of U-235 at a known activity level in the soil of about $7 \mathrm{pCi} / \mathrm{gram}$. Each point on each data plot represents an independent count. Count times of 3 minutes (Counts 1 -10), 10 minutes (Counts $11-15$ ), and 30 minutes (Counts 16-20) were used. A value of 0 in the plot (with no uncertainty bar) means that for that count, the nuclide was not identified by the analysis software. Note from Figures 6 and 7 that the output from Probe No. 2 is repeatable and reads about $1 \mathrm{pCi} / g r a m$ high. Probe No. 1 (with the lightguide) is not as repeatable and reads about $2 \mathrm{pCi} /$ gram low. We have demonstrated that the repeatability of Probe No. 1 can be improved by modifying the analysis software.

The energy of the main U-235 gamma emission is about $186 \mathrm{keV}$. To understand one contributing factor to the nonrepeatability of Probe No. 1, refer to Figure 8 which is a plot of detector counts versus gamma energy during a count in a soil sample containing $7 \mathrm{pCi} / \mathrm{gram}$ of U-235. The response of the Probe No. 2 (without lightguide) is shown by the solid line. Note that a peak can be observed in the solid line at the $186 \mathrm{keV} \mathrm{U}-235$ energy. Part of the area under the solid line is shaded between $50 \mathrm{keV}$ and $350 \mathrm{keV}$. The response of the Probe No. 1 assembly is shown by the dotted line. Note that in this case the background continuum is higher than the dotted curve, which makes the $186 \mathrm{keV}$ peak difficult to discern. By comparing the two response functions in Figure 8, it is apparent that the effect of the lightguide in Probe No. 1 is to reduce the signal-tonoise ratio of the detection system. The peaks contain about the same number of counts for both probes, but the peaks are lower and broader for Probe No. 1, making it more difficult for the analysis software to identify a peak.

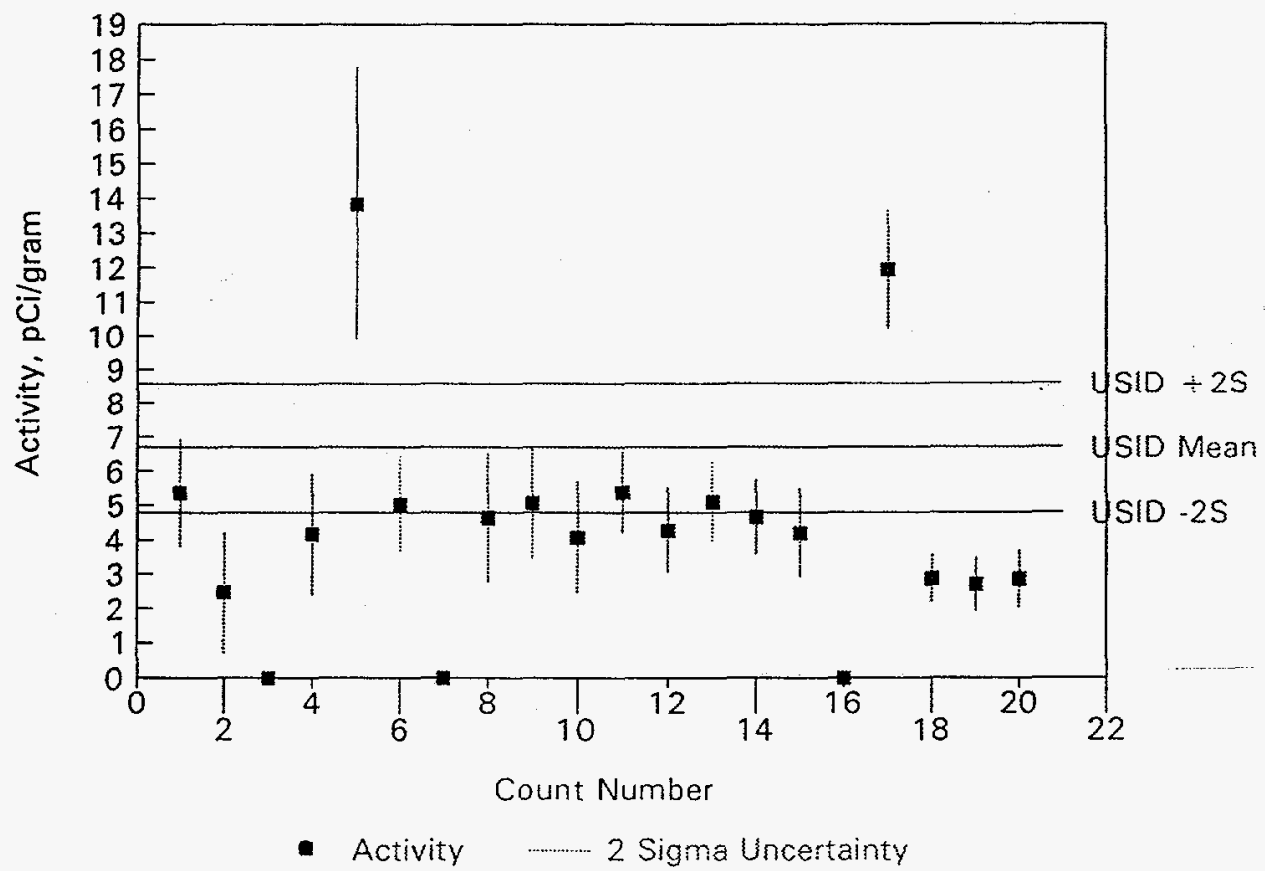

Fig. 6. Activity versus count number for Probe No. 1 (U-235 activity : Test D1E) 


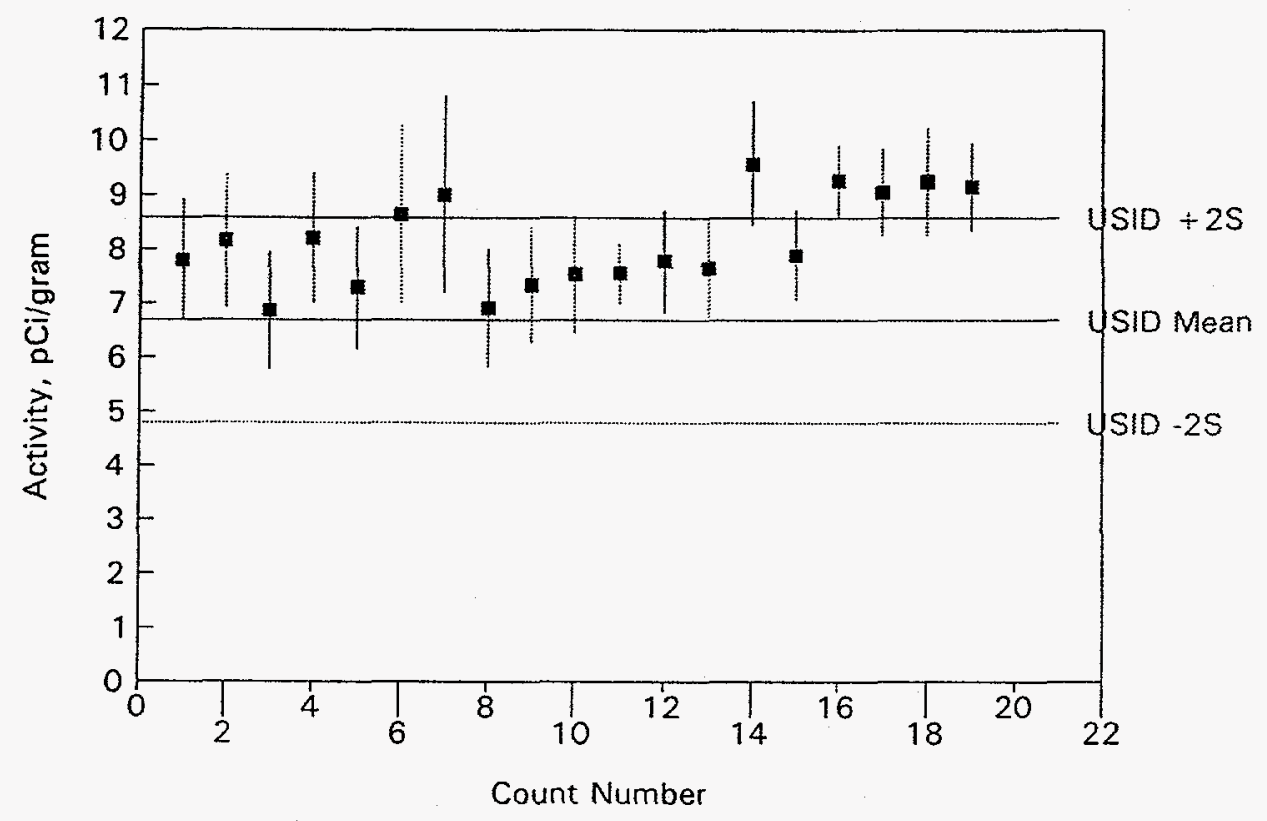

- Activity ....... 2 Sigma Uncertainty

Fig. 7. Activity versus count number for Probe No. 2 (U-235 activity : Test N1E)

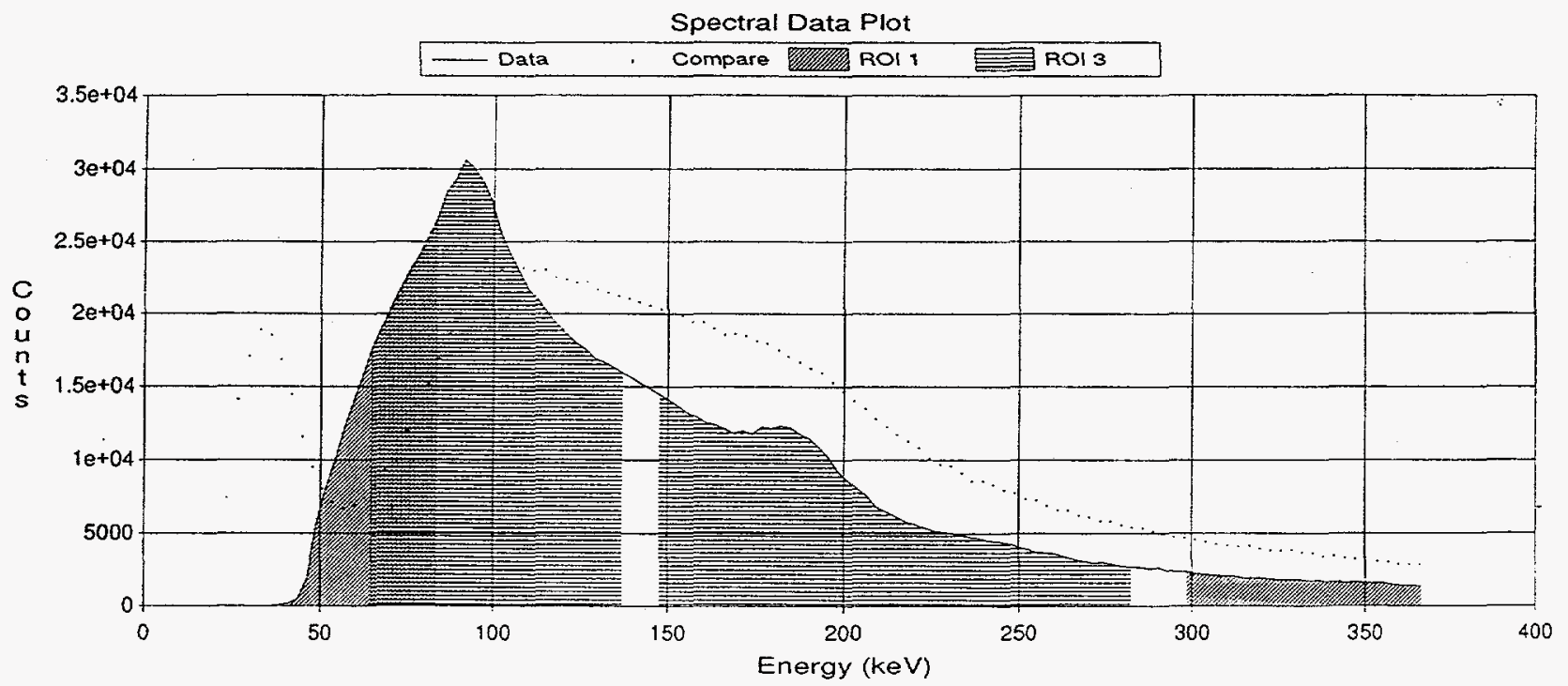

Fig. 8. Counts versus gamma energy for Probe No. 1 (dotted line) and Probe No. 2 (solid line) 


\section{CONCLUSION}

A probe was designed for in situ testing at a DOE radioactive waste site. The probe is based on a $10 \mathrm{~cm}^{2}$ cone penetrometer tool, incorporating a CsI(Tl) scintillator, butt-coupled PMMA lightguide, and detection electronics. The performance of this probe was estimated using a soil model for each of the radionuclides identified. The predicted performance was generally verified during a series of lab tests. In the field tests, we detected and quantified U-235 at activity levels less than $5 \mathrm{pCi} / \mathrm{gram}$ with count times of 10 minutes or less. We also detected U-238 at activity levels of less than $20 \mathrm{pCi} / \mathrm{gram}$ with count times of 10 minutes or less, and we detected and quantified U-238 at activity levels less than 45 pCi/gram with count times less than 30 minutes.

\section{ACKNOWLEDGMENTS}

This work was supported by the Morgantown (WV) Energy Technology Center for the Office of Technology Development (EM-50), U.S. Department of Energy, Contract Number DE-AC21-92MC29103. 